CERN-EP/87-104

5 June 1987

\title{
A SUPERCONDUCTING RADIOFREQUENCY COMPLEX FOR MOLECULAR, NUCLEAR AND PARTICLE PHYSICS
}

Ugo Amaldi ${ }^{1)}$

\author{
Presented at \\ Topical Seminar on Heavy Flavours \\ S. Miniato, May 25-29, 1987 \\ and \\ Meeting on New Projects in Particle and Nuclear Physics \\ Organized by the Italian National Institute for Nuclear Physics (INFN) \\ Rome, June 8-10, 1987 \\ To be published \\ in the Proceedings of the S. Miniato Seminar
}

1) CERN, EP Division, CH 1211 Genève 23, Switzerland 


\section{A SUPERCONDUCTING RADIOFREQUENCY COMPLEX FOR MOLECULAR, NUCLEAR AND PARTICLE PHYSICS}

Ugo AMALDI

CERN, EP Division, CH 1211 Genève 23, Switzerland

\section{INTRODUCTION}

Superconductivity applied to radiofrequency cavities is a fast developing technology ${ }^{1,2}$ at present being used for powering free electron lasers $(\mathrm{FEL})^{3}$, for constructing nuclear physics linear accelerators ${ }^{4}$, for accelerating electrons (and positrons) in circular machines ${ }^{2,5}$. Recently the fully superconducting colliding linear accelerators, which have been proposed long ago as means of achieving low energy electron-electron ${ }^{6}$ and high energy electron-positrons ${ }^{7}$ collisions, have been studied in detail and judged valid means of producing high luminosity electron-positron annihilations at $\mathrm{TeV}$ energies ${ }^{8,9}$. Moreover, superconducting (SC) cavities, with frequencies in the range $300-500 \mathrm{MHz}$, have been considered as devices for powering CLIC, the CERN Linear Collider which would use $30 \mathrm{GHz}$ copper cavities to accelerate electrons and positrons to $(1+1) \mathrm{TeV}^{10.11}$.

In the frequency range $350-3000 \mathrm{MHz}$ four (or five) cell niobium structures, having the typical spherical shape which avoids electron multipacting ${ }^{12}$, have today an average electric field of about $7 \mathrm{MV} / \mathrm{m}$ with a quality factor $\mathrm{Q} \simeq(3-5) 10^{9}$ at a temperature of $4 \mathrm{~K}^{1,2,13}$. The gradients of niobium cavities have been steadily improving during the last years and one can confidently predict that, by further $R \& D$, one may reach $20-30 \mathrm{MV} / \mathrm{m}$ at a temperature of $1.8 \mathrm{~K}^{8.9}$. The sputtering of a thin $\mathrm{Nb}_{3} \mathrm{Sn}$ (or $\mathrm{NbN}$ ) film on the inside of a copper cavity ${ }^{14}$ may lead to even larger fields, the theoretical limit being about $100 \mathrm{MV} / \mathrm{m}^{15}$. Thin films of the recently discovered warm superconductors, with their enormous critical magnetic field, deposited on a copper structure cooled at Helium temperatures, promise even larger gradients, but are technologically far into the future.

On October 8th, 1985, at the round table on 'Perspectives and value of frontier research in physics', organized at Trieste by the Italian Physical Society for the concluding session of its 72 th National Conference ${ }^{16}$, I proposed the idea of a laboratory which would use SC cavities

1. to produce intense beams of FEL infrared and millimeter electromagnetic radiation,

2. to accelerate continuous beams of electrons for nuclear physics experiments,

3. to build fully superconducting colliding linacs of electrons and positrons around $50+50$ $\mathrm{GeV}$ as intense factories of $Z^{\circ}$ bosons and of top and antitop quarks,

4. to develop the technologies needed for future $\mathrm{TeV}$ colliders.

As mentioned above, the first two programs are already under way in existing laboratories ${ }^{1-4}$. The third one was described at the CERN Accelerator School in Oxford ${ }^{17}$. The idea was to use the established properties of SC cavities to produce high luminosity and low energy $(\sim 100 \mathrm{GeV})$ electron-positron collisions with a very small energy spread $\left(\Delta \mathrm{E} / \mathrm{E}<10^{-3}\right)$. With the electric fields 
achievable today $(\sim 7 \mathrm{MV} / \mathrm{m})$ the total length of such a collider is more than $10 \mathrm{~km}$, probably too much for an intermediate size laboratory. The situation changed during 1986, when it became clear that intense sources of b-quarks would open new experimental possibilities, especially in view of the experimental motivations on the mixing of $B$ and $\bar{B}$ mesons presented by the UA1 Collaboration, later quantitatively proven to be unexpectedly large for the $B_{\alpha}$ system by the Argus experiment at DORIS $^{18}$. Following the Heidelberg meeting, where all these arguments were debated ${ }^{19}$ and a proposal for a circular beauty factory presented ${ }^{20}$, Guy Coignet and myself came to the conclusion ${ }^{21}$ that a beauty factory based on SC linacs with recirculators is very attractive because the small energy spread $\left(\Delta \mathrm{E} \simeq 10 \mathrm{MeV}\right.$ ) goes naturally together with a very large luminosities $\left(\mathrm{L} \geq 10^{33} \mathrm{~cm}^{-2}\right.$ $s^{-1}$ ). The conceptual design of such a SC linear beauty factory (together with its possibilities as source of FEL electromagnetic radiation, of continuous electron beams for nuclear physics and of synchrotron radiation) was presented at two dedicated meetings ${ }^{22}$ and published ${ }^{23}$.

In October 1986 a Discussion Meeting was organized in Frascati on 'SC Linear Accelerators' and the leading world experts in SC cavities, linear accelerators, high power FEL's and SC linear colliders were invited ${ }^{24}$. At the end of the discussion the frequency range $350-500 \mathrm{MHz}$ was indicated as best suited for such an accelerator complex. (This point will be taken up again in the next section.)

Taken all together, these facts prove that RF superconductivity is not only applicable to many fields of physics but is also rapidly developing. A physics laboratory, which would chose it as the unifying theme of its activity, would not only have first rate physics goals but could also contribute to very important technological developments.

An accelerator complex, of the type described in this report, based on SC cavities is within the technical and financial possibilities of a National Laboratory. In this report 1 describe the four successive stages through which the complex could be realized. For lack of time I devote only few lines to the physics objectives. The cost of the accelerator complex and some points of scientific policy will be touched upon in the last two sections.

\section{STAGE 1: FREE ELECTRON LASERS AND DC ELECTRON BEAMS FOR NUCLEAR PHYSICS}

The first stage is schematically drawn in Fig. 1. For high peak power FEL's the electron source has to have the possibility of running with a small emittance and a large peak current. Recent developments at Stanford University and Los Alamos are very encouraging ${ }^{25,26}$. At Los Alamos the RF source, shown in Fig. 2, runs at $1.3 \mathrm{GHz}$ and has a $\mathrm{Cs}_{3} \mathrm{Sb}$ photocathode which is illuminated by a neodymium laser. It has already produced more than $100 \mathrm{~A}$ of peak current with an invariant emittance $\epsilon_{\mathrm{n}} \simeq 10^{-5} \mathrm{~m}$ and a bunch length $\sigma_{\mathrm{z}} \simeq 30 \mathrm{~mm}$ for a total number of electrons/bunch $\mathrm{N}=4 \times 10^{10}$ and a brightness $\sim 10^{11} \mathrm{~A} / \mathrm{m}^{2}$. Further tests are going on with a postaccelerating cavity, which should produce directly $2 \mathrm{MeV}$ electrons. The first source is already satisfying the requirements of XUV-FEL's. The Los Alamos group thinks that the new source should give $\epsilon_{n} \simeq$ $2 \times 10^{-6} \mathrm{~m}$ at lower peak currents, corresponding to $\mathrm{N} \simeq 10^{10}$ electrons/bunch ${ }^{26}$. As we shall see in the next section, progress in this direction would allow a considerable saving in the further stages, because the emittance of the electron bunches would not need to be damped in magnetic rings. 


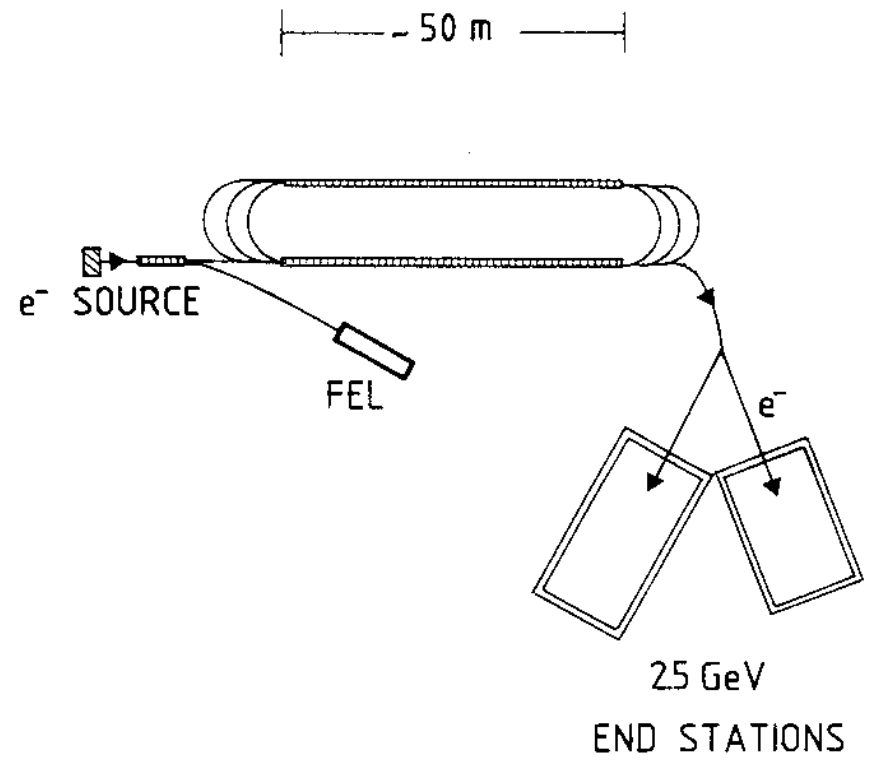

FIGURE 1

Scheme of stage 1. The electron sources needed for FEL and nuclear physics have different characteristics, as explained in the text.

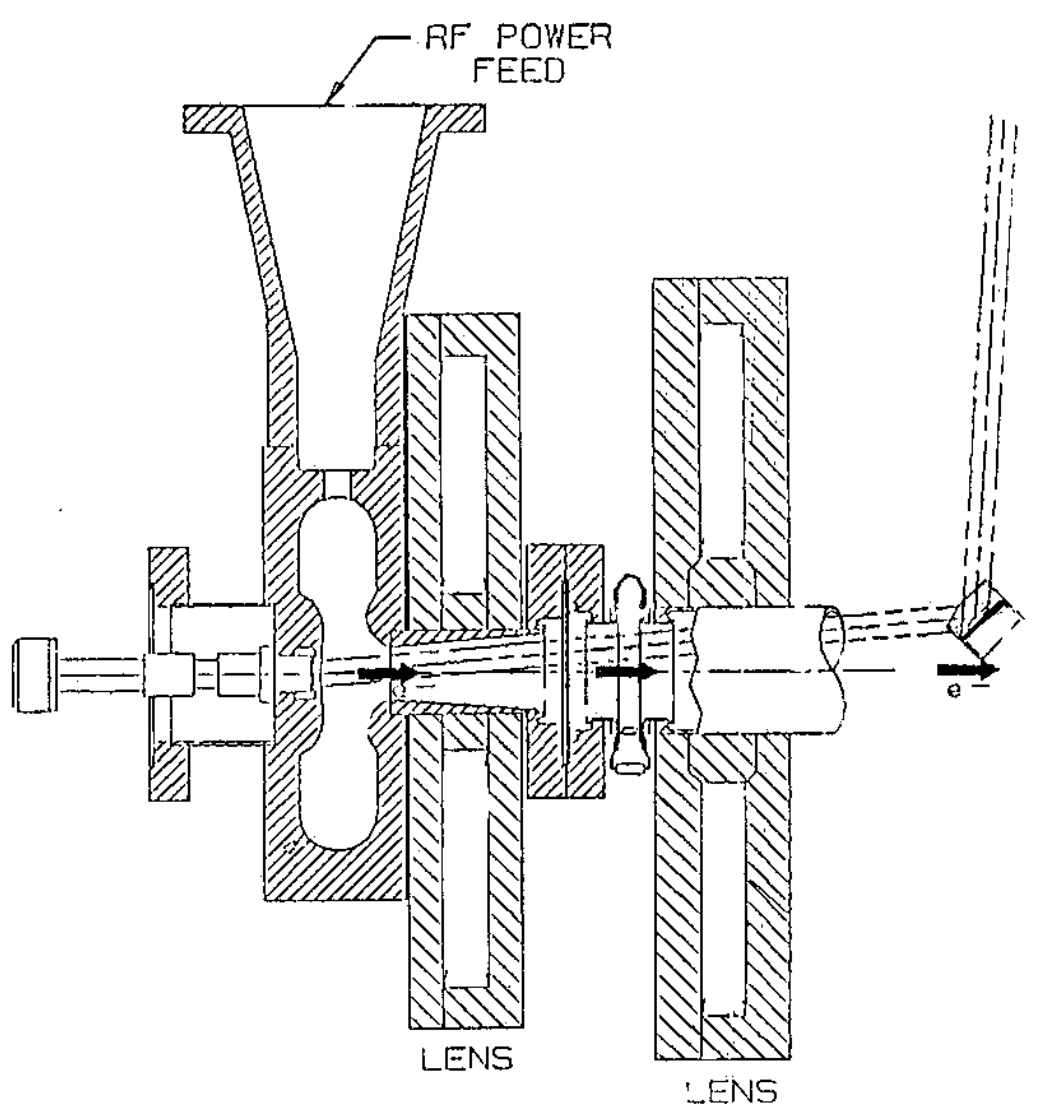

FIGURE 2

High-brightness electron source developed at Los Alamos ${ }^{26}$. 
Figure 1 shows a FEL ondulator placed after a preacceleration of the electron bunches to about $50 \mathrm{MeV}\left(\gamma \simeq 10^{2}\right)$. Other FEL's could of course either run in parallel, by making use of the infrastructures of the laboratory, or be feed at higher energies, by using the bunches further downstream in the main accelerator.

For nuclear physics studies CW beams are preferred, so that a low peak current source capable of filling all the RF buckets is sufficient. To serve more end stations with electron beams, which appear practically continuous to fast coincidence detectors, high frequencies are more advantageous. CEBAF has chosen the Cornell frequency $f_{R F}=1.5 \mathrm{GHz}$. FEL and collider applications, on the other end, tend to favour low frequency because in this case the stored energy, being proportional to $f_{R F}{ }^{-2}$, is larger and transverse wake field effects (proportional to $f_{R F}^{3}$ ), are smaller. As anticipated in the Introduction, the Frascati Discussion Meeting concluded that the frequency of a SC radiofrequency complex has to be in the range 350-500 MHz. After the Meeting it was agreed that $\mathrm{f}_{\mathrm{RF}}=500 \mathrm{MHz}$ is a good choice for the present application, also in view of the fact that $350 \mathrm{MHz}$ cavities are quite large transversally.

As shown in Fig. 1, the $2.5 \mathrm{GeV}$ are obtained in the scheme proposed by G.Coignet and myself by using recirculators at the end of each linac. CEBAF uses three and four respectively. The optimum number and the location of the recirculators has to be optimized once the site and the cost of the various components are well known. Assuming $7 \mathrm{MV} / \mathrm{m}$ as average field and a length of $\sim 50 \mathrm{~m} / \mathrm{linac}$, the two end stations can be served with $\geq 200 \mu \mathrm{A}$ of electron average current at energies $E \leq 2.5 \mathrm{GeV}$; the final energy could be higher if either electric fields larger than $7 \mathrm{MV} / \mathrm{m}$ can be produced or a larger linac is built. The physics potentialities of such a beam have been widely presented in connection with the CEBAF and the ALS project in Saclay, have been summarized in the proceedings of the Frascati Meeting ${ }^{24}$ and in recent reviews ${ }^{4}$, and are not presented here in detail. The main points are:

i) Experiments on single nucleon emission of the type $\left(e, e^{\prime} p\right),\left(e, e^{\prime} n\right),\left(e, e^{\prime} \alpha\right)$;

ii) Studies of two nucleon correlations with the reactions $\left(e, e^{\prime} 2 N\right)$;

iii) Measurements of the neutron and deuteron form factors (with polarized targets and beams);

iv) Search for effects of new degrees of freedom in nuclei. (This may require $E>5 \mathrm{GeV}$ );

v) Production of nuclear resonances, e.g. (e, $\left.e^{\prime} p \pi\right)$ reactions;

vi) Experiments devoted to careful measurements of parity violation.

For most of this program, an energy in the range $2.5-3.0 \mathrm{GeV}$ is sufficient. The data collected at this first stage would form a good 'classical' basis to compare with the ones obtained at the further $10 \mathrm{GeV}$ stage with energies definetely larger than those available at CEBAF.

In the field of Free Electron Lasers, superconducting cavities running continously offers the possibility of combining high peak powers with large average powers. Such power can be used for studies of molecular physics and chemistry. Note that around $\lambda \simeq 1 \mathrm{~mm}$ a CW Free Electron Laser could deliver megawatt of power to heat a fusion plasma; the technique could be tested at the SC complex. 


\section{STAGE 2: CHARM AND TAU PHYSICS}

The interest in high luminosity electron-positron annihilation in the range $E_{\mathrm{cm}} \simeq 3-5 \mathrm{GeV}$ has been often discussed ${ }^{20}$ and even proven by the many interesting results produced by MARK ill at SPEAR, which at present runs at $L \simeq 2 \times 10^{30} \mathrm{~cm}^{-2} \mathrm{~s}^{-1}$. Recently the richness of $\tau \bar{\tau}$ physics has also been underlined ${ }^{27}$. The ring being built in Beijing will reach $2 \times 10^{31} \mathrm{~cm}^{-2} \mathrm{~s}^{-1}$.

The present proposal aims at $\mathrm{L} \simeq 2 \times 10^{32} \mathrm{~cm}^{-2} \mathrm{~s}^{-1}$ by colliding only once electron and positron bunches. This opens the door to unique physics in the following domains:

i) Searches of glueballs and light Higgs particles;

ii) Measurement of the mass of $v_{\tau}$ (the sensitivity is $\approx 1 \mathrm{MeV}$ );

iii) Limit on second class currents in $\tau$-decay;

iv) Measurements of the Cabibbo angle in $\tau$-decay;

v) Limits on $D^{\circ}-\bar{D}^{\circ}$ mixing (theoretically one expects $<10^{-4}$ );

vi) Measurements of the elements $U_{c s^{\prime}} U_{c d}$ of the Cabibbo-Kobayashi-Maskawa matrix;

vii) Search for flavour changing neutral currents to a level of $10^{-7}$, and so on.

A possible scheme of a $c \bar{c}$ and $\tau \bar{\tau}$ factory is shown in Fig. 3. Damping rings are used to reduce the emittance of the positron bunches, which are continously injected and extracted after $\sim 15 \mathrm{~ms}$ of waiting time.

To produce positrons, one can resort to a low energy ( $\sim 200 \mathrm{MeV})$ high current SC specialized linac. Figure 3 shows the scheme based on a such low energy linac. A more elegant solution makes

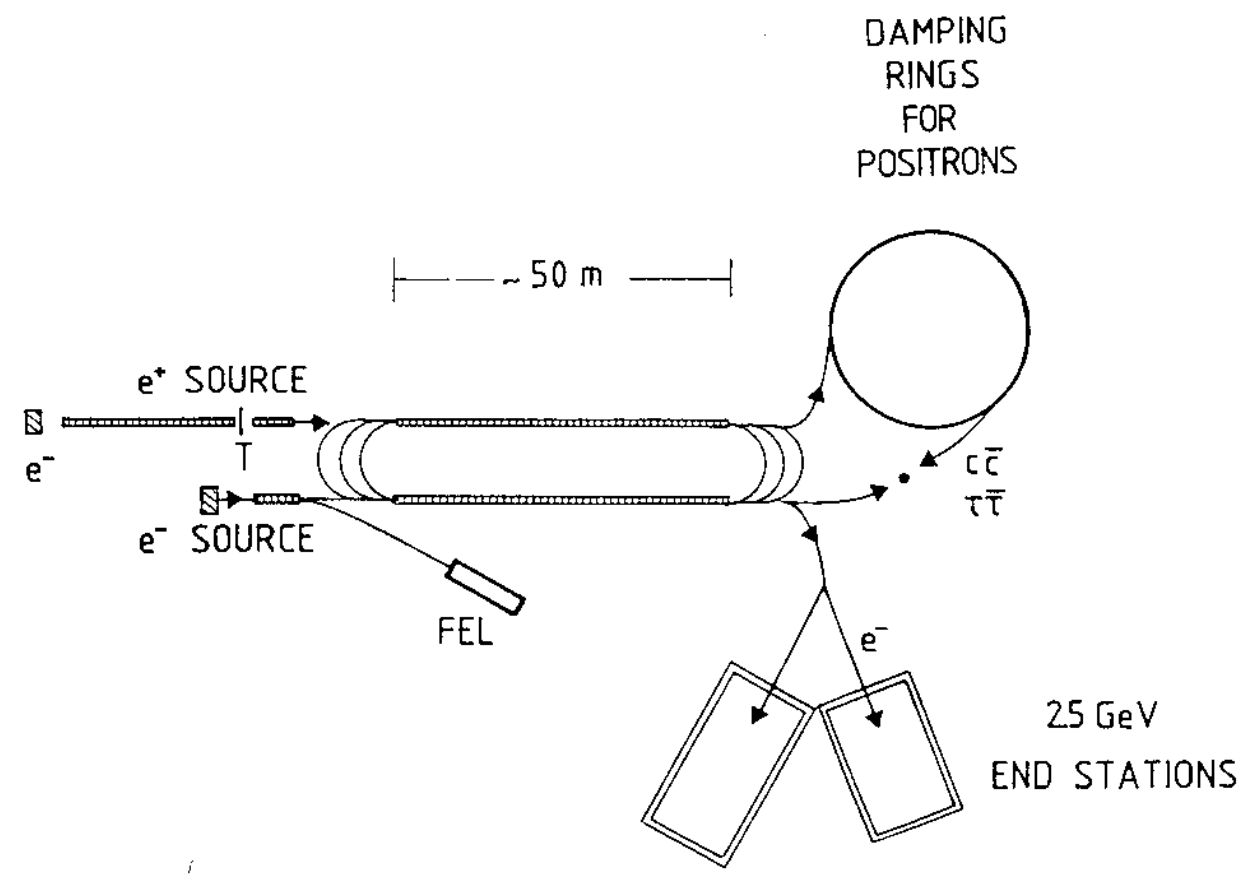

FIGURE 3

Schematic drawing of stage 2. The positrons are supposed to be obtained by collisions of about $200 \mathrm{MeV}$ electrons on a heavy target. In the text another possibility is discussed: the target $T$ is illuminated with the electrons accelerated at $2 \mathrm{GeV}$ in the racetrack linac. The positron damping rings have a circumference $2 \pi R \simeq 100 \mathrm{~m}$ and there are at least four of them. If a low-emittance electron source cannot be built, also the electron bunches have to be damped in dedicated rings. 
use of electrons of higher energies. After being accelerated by the racetrack linac to about $2 \mathrm{GeV}$, bunches of $N \simeq 4 \times 10^{11}$ electrons having a large emittance $\left(\epsilon_{n} \geq 10^{-4} \mathrm{~m}\right)$ impinge on a heavy target. Low energy positrons are collected and then accelerated to $-50 \mathrm{MeV}$ before injection in the racetrack linac. The efficiency of present positron sources are such that about $20 \mathrm{GeV}$ have to be spent to get a positron, so that the electron intensity is sufficient to get more than $\mathrm{N} \simeq 4 \times 10^{10}$ positrons/bunch. At $50 \mathrm{MeV}$ the positron invariant emittance will be $\epsilon_{\mathrm{n}}=10^{-2}-10^{-3} \mathrm{~m}$, and has to be damped in the rings after acceleration to the required final energy ( $1.5 \leq E \leq 2.5 \mathrm{GeV})$.

Large peak and average powers have to be dissipated in the positron target; the peak power is equal to the one foreseen in the SLC target, and thus the temperature shock of a single bunch is not a problem. For a repetition frequency $f_{r}=7 \mathrm{kHz}$ the average beam power is $\sim 1 \mathrm{MW}$, about $1 / 3$ of which has to be dissipated in the target. This is technologically possible, as shown by the design and construction of spallation sources, but certainly difficult and represent the biggest challenge of the whole superconducting complex. (SLC beam has an energy of $33 \mathrm{GeV}$, the same peak power but an average power $\sim 0.05 \mathrm{MW}$.) The production and acceleration of $4 \times 10^{11}$ electrons is also at the limit of space charge and wake fields effects, but the SLAC experience with bunches of $5 \times 10^{10}$ electrons in a structure which has a wavelength 10 times smaller proves that it is feasible. Moreover, CERN will be developing such sources in the framework of the studies for CLIC ${ }^{28}$.

Table 1 contains a possible list of beam parameters at the collision point. The first column is taken from Ref. 23 and is computed by using approximate analytic expressions ${ }^{11,29-31}$. Since then the program written by Yokoya ${ }^{30}$ has been used by P.T. Cox to compute the parameters. This simulation program describes the colliding particles as ensembles of superparticles and follows their path and radiation during the collision. The program uses as input the bunch energy $E$, the number of particles per bunch $N$, the invariant emittance $\epsilon_{n}$, the $\beta$-value at the crossing point $\beta^{*}$ (so that the transverse r.m.s. radius is $\left.\sigma_{\mathrm{t}}=\sqrt{\epsilon}_{\mathrm{n}} \beta^{*} / \gamma\right)$ and the r.m.s. length of the bunch $\sigma_{\mathrm{z}}$. It can be seen from the Table that there is good agreement between the approximate equations and the results of the detailed simulation.

In Table $1, \Upsilon$ is the ratio between the average critical energy $E_{c}$ of the photons radiated in the collision and the beam energy $E$. I underline that this collider, as the others described in the next sections, would run in the so called 'classical regime', since the ratio of the photon critical energy to the bunch energy is $\Upsilon<<1$. By $\langle\epsilon\rangle$ I indicate the fractional energy lost by the particles in this 'beamstrahlung' process and by $\mathrm{N}_{\gamma}$ the average number of photons radiated per particle. The fractional r.m.s. spread of the c.m. energy $\mathrm{W}$ due to beamstrahlung is indicated by $\sigma_{w^{\prime}}$ and $\Delta \mathrm{W}=\sigma_{\mathrm{w}}$ $W$. Since the distribution of the c.m. energy $W$ caused by the beamstrahlung radiation is not normal, the fractional r.m.s. value $\sigma_{w}$ has a not well defined meaning and it should not be forgotten, when considering the numerical values of the parameter $\sigma_{w}$ and $\Delta \mathrm{W}$, that the distribution is not gaussian.

The quantity $D$ of Table 1 is the 'disruption parameter', which measures the focusing effect that one beam produces on the other ${ }^{32}$. For $D \geq 1 / 2$ pinch effects become important and the luminosity is enhanced by the factor $\mathrm{H}$, which in Ref. 23 was taken to be $6^{32}$; the detailed simulation program run for these particular beam conditions confirm it to be $\mathrm{H}=6.3$. 
Table 1

Parameters for electron-positron collisions around $(2+2) \mathrm{GeV}$

\begin{tabular}{|c|c|c|c|}
\hline Quantity & Symbol/Unit & Ref. 23 & $\begin{array}{l}\text { Yokoya } \\
\text { Program }^{30}\end{array}$ \\
\hline bunch energy & $\mathrm{E} / \mathrm{GeV}$ & $1.5-2.5^{*}$ & \\
\hline luminosity & $\mathrm{L} / \mathrm{cm}^{-2} \mathrm{~s}^{-1}$ & $2 \times 10^{32}$ & $2 \times 10^{32}$ \\
\hline power/beam & $\mathrm{P} / \mathrm{MW}$ & 0.13 & \\
\hline bunch average frequency & $\mathrm{f}_{\mathrm{r}} / \mathrm{kHz}$ & 7 & \\
\hline invariant emittance & $\epsilon_{\mathrm{n}} / \mathrm{m}$ & $2 \times 10^{-6}$ & \\
\hline disruption parameter & $\mathrm{D}$ & 17 & \\
\hline pinch factor & $\mathrm{H}$ & 6 & 6.3 \\
\hline particles/bunch & $N$ & $4 \times 10^{10}$ & \\
\hline bunch r.m.s. length & $\sigma_{\mathrm{z}} / \mathrm{mm}$ & 1.5 & \\
\hline$\beta$-value at IP & $\beta^{*} / \mathrm{mm}$ & 5 & \\
\hline bunch r.m.s. radius & $\sigma_{\mathrm{t}} / \mu \mathrm{m}$ & 1.6 & \\
\hline av. critical energy & $\mathrm{E}_{\mathrm{c}} / \mathrm{MeV}$ & 0.17 & \\
\hline$E_{c} / E$ & $\Upsilon$ & $8.5 \times 10^{-5}$ & $1.1 \times 10^{-4}$ \\
\hline fractional energy loss & $<\epsilon>$ & $4.7 \times 10^{-5}$ & $3.7 \times 10^{-5}$ \\
\hline av. number of photons & $\mathrm{N}_{\gamma}$ & 1.4 & 1.1 \\
\hline collision energy & $\mathrm{W} / \mathrm{GeV}$ & 4.0 & \\
\hline fract. r.m.s. of W & $\sigma_{w}$ & $4.5 \times 10^{-5}$ & $4.4 \times 10^{-5}$ \\
\hline r.m.s. of $W=\sigma_{w} W$ & $\Delta \mathrm{W} / \mathrm{MeV}$ & 0.1 & 0.1 \\
\hline
\end{tabular}

*) The other parameters are given for $E=2.0 \mathrm{GeV}$.

I note that, on purpose, the parameters $\sigma_{z^{\prime}} \beta^{*}$ and $\sigma_{t}$ chosen for the collider are similar to the SLC ones $^{33}$. (This seems today the only possible basis for a design. In one year from now, when more will be known on the production of micron bunches, one may revise this choice and, possibly, get for this collider and the ones presented in the next sections even larger luminosities.) We have instead chosen an invariant emittance which is ten times smaller than for SLC, to compensate the reduction in the energy of the bunches, and a disruption parameter which is ten times bigger. The bunch frequency is hundred times larger than in SLC, as allowed without difficulty by the use of SC cavities which run continuously.

The computed energy spread $\sigma_{w}$ due to beamstrahlung is completely negligible, so that the only source of c.m. energy spread is the energy spread of the positron and electron bunches. Positron bunches have their emittance damped in special magnetic rings down to $\epsilon_{\mathrm{n}} \simeq 2 \times 10^{-6} \mathrm{~m}$, which is what can be obtained today in the best synchrotron light sources, and the energy spread is usually $\Delta E / E<10^{-3}$, i.e. $\Delta E<2 \mathrm{MeV}$. I want to underline that, if the electron source cannot give directly such a small emittance, together with a small enough bunch length and energy spread after proper 
rotation in the longitudinal phase space, also the electron bunches have to be stored in damping rings and the cost of the damping ring system doubles.

The required invariant emittance $\left(\epsilon_{\mathrm{n}} \simeq 2 \times 10^{-6} \mathrm{~m}\right)$ is small and optimized damping rings (DR) are needed. In Ref. 23 we have chosen as nominal damping energy $\mathrm{E}_{d}=2.5 \mathrm{GeV}$ to reduce the effect of intrabeam scattering and have short damping times ${ }^{23}$. By following the work done in connection with synchrotron light sources, we used the formulae which are valid for lattices of the Chasman-Green type ${ }^{34}$, each module being formed by two bending magnets and five quadrupoles:

$$
\begin{aligned}
& \epsilon_{\mathrm{d}} /[\mathrm{m}] \simeq 1.2 \times 10^{-11}\left(\gamma_{\mathrm{d}} / \mathrm{N}_{\mathrm{d}}\right)^{3} \\
& \tau /\left[\mathrm{s} \cdot \mathrm{T}^{2}\right] \simeq 30 /\left(\gamma_{\mathrm{d}} \mathrm{B}_{\mathrm{d}}{ }^{2}\right)
\end{aligned}
$$

where $\epsilon_{d}$ is the equilibrium emittance, $\gamma_{d}$ is the $\gamma$-factor of the particles of energy $E_{d}$ in the DR's, $\tau$ is the damping time, $N_{d} / 2$ is the number of achromatic modules per ring, and $B_{d}$ is the field in the bending magnets. At $E_{d}=2.5 \mathrm{GeV}$, to get an equilibrium emittance $\epsilon_{d} \simeq 10^{-6} \mathrm{~m}$ the above equation implies $N_{d} \simeq 100$ magnets. With $B_{d}=1.6 \mathrm{~T}$ the damping time is $\tau \simeq 2.5 \times 10^{-3} \mathrm{~s}$. (At $E_{d}=$ $2.0 \mathrm{GeV}$ with $\mathrm{B}_{\mathrm{d}}=1.3 \mathrm{~T}$, Eqs. (1) give $\epsilon_{\mathrm{d}} \simeq 2 \times 10^{-6} \mathrm{~m}$ and $\tau \simeq 4.7 \mathrm{~ms}$.) The radius of curvature is $\varrho_{d} \simeq 5 \mathrm{~m}$, the average radius $\mathrm{R}=15 \mathrm{~m}$ and the length of each bending magnet is about $30 \mathrm{~cm}$, similar to the length of the magnets used in the FODO rings built for the SLC collider ${ }^{33}$.

One can now compute the total number of damping rings. The ratio of the total length of the positron DR's $\ell_{d}$ to the average distance betweenthe bunches $\ell_{b}$ in each DR is ${ }^{31}$.

$$
\ell_{\mathrm{d}} / \ell_{\mathrm{b}}=(\mathrm{t} / \tau) \mathrm{f}_{\mathrm{r}} \tau \simeq 160
$$

where $t$ is the bunch waiting time in a $D R$, and we have taken $(t / \tau) \simeq 5, \tau=4.7 \mathrm{~ms}$ and $\mathrm{f}_{\mathrm{r}}=7 \mathrm{kHz}$. By putting in the DR's trains of 5 bunches at a distance $\lambda_{\mathrm{RF}}=60 \mathrm{~cm}$ ( $f_{\mathrm{RF}}=500 \mathrm{MHz}$ ) separated by about $10 \mathrm{~m}$, from Eq. (2), $\ell_{\mathrm{b}} \simeq 2.5 \mathrm{~m}$ and $\ell_{\mathrm{d}} \simeq 400 \mathrm{~m}$. (This needs the development of very fast kickers.) To achieve the needed $f_{r}=7 \mathrm{kHz}$ frequency, for damping $2 \mathrm{GeV} \mathrm{e}{ }^{+}$bunches one has to built at least 4 rings, each having a circumference $2 \pi R=100 \mathrm{~m}$. (Maybe two, or more, rings can be put one on top of the other by using the same iron for the return yoke, as done in the CERN Booster.) At any moment each ring contains $8 \times 5=40$ bunches and a refined feedback system is required for damping the multibunch instabilities. Trains of bunches are useful to reduce the overall length of the damping rings, but may cause problems in the final focus system. The disrupted bunches may perturb the incoming bunches before interaction and reduce luminosity. The minimum distance at which the bunches of the same train can sit needs a careful design of the final focus system with its separator, which does not exist yet. The results of such a study could be an increased number of damping rings.

The positrons (electrons) radiate in each ring $\sim 0.25 \mathrm{MW}$ of average power. The critical energy of the radiation produced in the bending magnets is $3.5 \mathrm{keV}$. Clearly each ring is a high-brightness and powerful source of wide band synchrotron radiation. Wigglers could be added to have a tunable and narrow band source. In conclusion, it is possible to use the DR's as synchrotron radiation sources, but to be more precise one needs a careful optimization of their design, including the computation of the dynamical aperture with and without wiggler magnets. 


\section{STAGE 3: BEAUTY FACTORY AND 10 GEV NUCLEAR PHYSICS}

By increasing the positron energy to $2.5 \mathrm{GeV}$ the same damping ring system has a damping time $\tau=2.5 \mathrm{~ms}$ and can produce bunches at the average repetition frequency $\mathrm{f}_{\mathrm{r}} \approx 12 \mathrm{kHz}$. Following the scheme described in the last section, this is obtained with 5 bunches per train and a train frequency $f_{t}=2.4 \mathrm{kHz}$; as before, 600 trains per second have to be extracted in succession from each of the four DR's. The bunches are accelerated in the second racetrack linac shown in Fig. 4. Electron bunches, either extracted from a dedicated DR system or directly coming from the $2.5 \mathrm{GeV}$ linac, are also accelerated up to $10 \mathrm{GeV}$. The two arches and the final focus system are very similar to the ones built for $\mathrm{SLC}^{33}$. They may also be used to rotate the bunches in the longitudinal phase space.

In the scheme shown in Fig. 4 we foresee to have electrons and positrons circulating in opposite directions both in the first and in the second acceleration racetrack linac. The counterrotating bunches have to be electrostatically separated in the arcs of the second linac. Moreover, quadrupole focusing has to be provided along the SC linacs and it could be that further calculations show that an easier solution is found when the two beams circulate in the same direction. This is a detail which does not modify our considerations.

For the $\Upsilon(4 S)$ studies one must aim at a luminosity $L=10^{33} \mathrm{~cm}^{-2} \mathrm{~s}^{-1}$. In Ref. 23 we have chosen a final beam current comparable to what will be produced at the CEBAF accelerator. Since CEBAF will produce $200 \mu \mathrm{A}$ at $4 \mathrm{GeV}$ with four recirculators, we assume similar average currents. However, our peak current is equal to the SLC one and $\sim 10^{4}$ times larger than at CEBAF.

To run in the high-resolution mode on the $\Upsilon$ (4S) peak (at $10576 \mathrm{MeV}$ ), which at half height is about $25 \mathrm{MeV}$ wide, one needs an energy spread smaller than $10 \mathrm{MeV}$. This implies a very small average beamstrahlung fractional energy loss by the $\mathrm{e}^{+ \text {' }} \mathrm{s}$ and $\mathrm{e}^{- \text {'s }} \mathrm{s}$ bunches: $\langle\epsilon\rangle \simeq 5 \times 10^{-4}$ at a beam energy $\mathrm{E} \simeq 5 \mathrm{GeV}$. In the 'continuum' the collider would run in the low resolution-high energy mode, for which one can instead accept $\langle\epsilon\rangle \simeq 10^{-2}$.

The values of the important parameters for both modes of running are collected in Table 2. Approximate expressions, which take into account the pinch effect, had been used in Ref. 23 to compute the values appearing in the second column of Table 2. The third column has been computed by P.T. Cox with the simulation program written by Yokoya ${ }^{30}$. Here again we can see that the analytic formulae are a good approximation to the numerical calculation.

In the high luminosity-low resolution mode the emittance and the frequency are equal to the ones needed in the high resolution mode, while the other parameters are somewhat more demanding. In particular the bunch length is $\sigma_{\mathrm{z}}=0.4 \mathrm{~mm}$ (instead of $1.3 \mathrm{~mm}$ ) and the $\beta$-function at the interaction point has the value $\beta^{*}=4.0 \mathrm{~mm}$ (instead of $6.0 \mathrm{~mm}$, as at SLC). This is feasible with SmCo permanent quadrupoles in view of the small energy spread achievable with SC cavities $(\triangle \mathrm{E} / \mathrm{E}$ $<10^{-3}$ ), which greatly reduces the problems connected with the chromaticity of the final focus system and the needed aperture to contain the disrupted beam ${ }^{35}$, whose angle is of the order of $\mathrm{D}^{1 / 2} \sigma_{\mathrm{t}} / \sigma_{\mathrm{z}} \approx 6 \times 10^{-3} \mathrm{rad}$.

The train operation of the rings implies that in the second acceleration stage $5 \mathrm{e}^{-}$and $5 \mathrm{e}^{+}$ bunches have to be accelerated from $2.5 \mathrm{GeV}$ to a maximum of $=10 \mathrm{GeV}$. In the high resolution mode the energy extracted by such a train is $0.3 \mathrm{~J} / \mathrm{m}$, to be compared with a stored energy of 45 

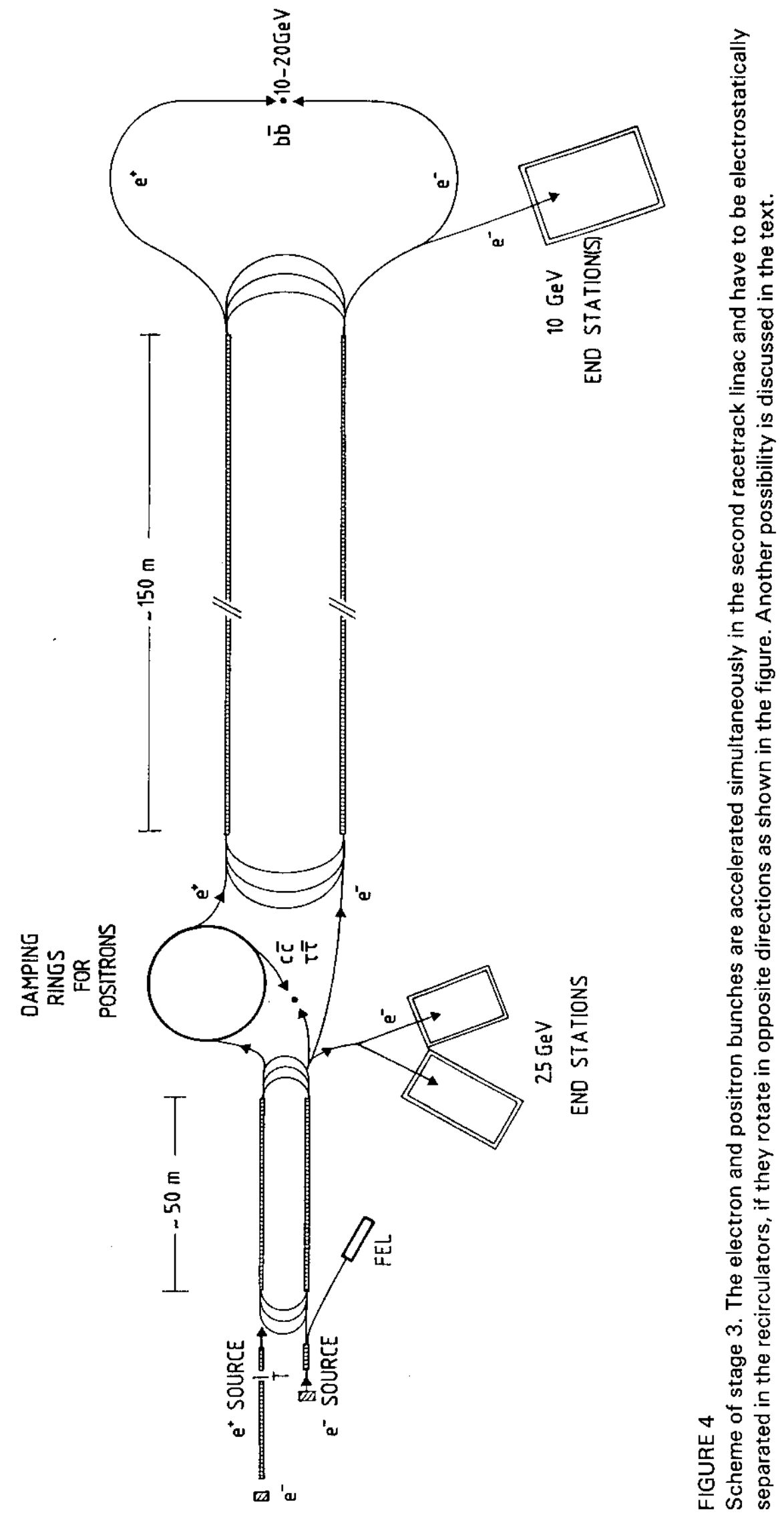
Table 2

Parameters for the high resolution and low resolution modes of the beauty factory

\begin{tabular}{|c|c|c|c|c|}
\hline \multirow[t]{2}{*}{ Symbol/Unit } & \multicolumn{2}{|c|}{$\begin{array}{l}\text { High resolution } \\
\text { mode }\end{array}$} & \multicolumn{2}{|c|}{$\begin{array}{c}\text { Low Resolution } \\
\text { mode }\end{array}$} \\
\hline & Ref. 23 & Yokoya ${ }^{30}$ & Ref. 23 & Yokoya $^{30}$ \\
\hline $\mathrm{E} / \mathrm{GeV}$ & $5-6^{*}$ & & $6-10^{*}$ & \\
\hline $\mathrm{L} / \mathrm{cm}^{-2} \mathrm{~s}^{-1}$ & $10^{33}$ & $1.3 \times 10^{33}$ & $10^{34}$ & $10^{34}$ \\
\hline $\mathrm{P} / \mathrm{MW}$ & 0.5 & & 1.5 & \\
\hline $\mathrm{f}_{\mathrm{r}} / \mathrm{kHz}$ & 12 & & 12 & \\
\hline$\epsilon_{\mathrm{n}} / \mathrm{m}$ & $2 \times 10^{-6}$ & & $2 \times 10^{-6}$ & \\
\hline $\mathrm{D}$ & 16 & & 13 & \\
\hline$H$ & 6 & 6.4 & 6 & 6.1 \\
\hline$N$ & $5 \times 10^{10}$ & & $8 \times 10^{10}$ & \\
\hline$\sigma_{\mathrm{z}} / \mathrm{mm}$ & 1.3 & & 0.4 & \\
\hline$\beta^{*} / \mathrm{mm}$ & 6.0 & & 4.0 & \\
\hline$\sigma_{\mathrm{t}} / \mu \mathrm{m}$ & 1.1 & & 0.60 & \\
\hline $\mathrm{E}_{\mathrm{c}} / \mathrm{MeV}$ & 2.3 & & 85 & \\
\hline$\Upsilon$ & $4.5 \times 10^{-4}$ & $5.6 \times 10^{-4}$ & $8.5 \times 10^{-3}$ & $1.0 \times 10^{-2}$ \\
\hline$\langle\epsilon>$ & $4.5 \times 10^{-4}$ & $3.6 \times 10^{-4}$ & $2.5 \times 10^{-2}$ & $1.8 \times 10^{-2}$ \\
\hline $\mathrm{N}_{\gamma}$ & 2.5 & 2.1 & 7.5 & 5.9 \\
\hline $\mathrm{W} / \mathrm{GeV}$ & $10-12$ & & $12-20$ & \\
\hline$\sigma_{w}$ & $3.5 \times 10^{-4}$ & $3.2 \times 10^{-4}$ & $1.3 \times 10^{-2}$ & $1.0 \times 10^{-2}$ \\
\hline$\Delta \mathrm{W} / \mathrm{MeV}$ & 3.4 & 3.2 & 250 & 200 \\
\hline
\end{tabular}

*) The other parameters are given for $E=5 \mathrm{GeV}$ and $\mathrm{E}=10 \mathrm{GeV}$ respectively.

$\mathrm{J} / \mathrm{m}$ at $7 \mathrm{MV} / \mathrm{m}$, if the frequency is $500 \mathrm{MHz}$. This corresponds to an energy difference between the first and the fifth $\mathrm{e}^{+} \mathrm{e}^{-}$collision of $\sim 15 \mathrm{MeV}$, still acceptable. From this point of view higher frequencies are not favored, but could be chosen if the number of bunches per train is smalier than 5 and the length of the DR's is increased. For completeness we note that the energy spread due to the finite length of each bunch when it is accelerated on the crest of the wave is completely negligible (less than $1 \mathrm{MeV}$ at $500 \mathrm{MHz}$ ).

As indicated in Fig. 4, the $10 \mathrm{GeV}$ continuous electron (and positron) beam can be sent to a high energy end station for experiments which are at the most interesting frontier between nuclear and particle physics. Total power is the main limitations to the achievable current, since $200 \mu \mathrm{A}$ correspond to $2 \mathrm{MW}$ beam power. This implies that more experiments can be certainly served at the same time. 


\section{STAGE 4: $Z^{\circ}$ AND TOPONIUM FACTORIES AND BEYOND}

The damping ring system is in itself a very interesting development in view of the construction of future TeV linear electron-positron colliders. Not by chance, the invariant emittance and the repetition frequency required by CLIC (the CERN Linear Collider) are equal to the ones used in our SC accelerator complex: $\epsilon_{\mathrm{n}}=2 \times 10^{-6} \mathrm{~m}$ and $\mathrm{f}_{\mathrm{r}}=6 \mathrm{kHz}^{28}$. As mentioned in the introduction, $\mathrm{SC}$ cavities can be used to power very high frequency copper structures to large accelerating fields ${ }^{10}$. Such an accelerator will not have a good energy resolution, but it could be used to abundantly produce $\mathrm{Z}^{\circ} \mathrm{s}$ at $(46+46) \mathrm{GeV}$, since the width of the intermediate vector boson is about $2 \mathrm{GeV}$. The length of such a linear collider depends on the available gradient. For $100 \mathrm{MV} / \mathrm{m}$ one would need $(500+500) \mathrm{m}$.

As shown in the second column of Table 3, the parameters are such that, using the positron (electron) source of the previous stages, at the $Z^{\circ}$ peak one could reach a luminosity which is more

Table 3

Parameters for $(50+50) \mathrm{GeV}$ collisions

\begin{tabular}{|c|c|c|}
\hline Symbol/Unit & $\begin{array}{l}\text { Low resolution collider } \\
\qquad\left(\mathrm{Z}^{\circ} \text {-factory }\right)\end{array}$ & $\begin{array}{l}\text { High resolution collider } \\
\text { (toponium-factory) }\end{array}$ \\
\hline $\mathrm{E} / \mathrm{GeV}$ & 50 & 50 \\
\hline $\mathrm{L} / \mathrm{cm}^{-2} \mathrm{~s}^{-1}$ & $2.5 \times 10^{33}$ & $2 \times 10^{32}$ \\
\hline P/MW & 2.0 & 1.0 \\
\hline $\mathrm{f}_{\mathrm{r}} / \mathrm{kHz}$ & 12 & 12 \\
\hline$\epsilon_{n} / m$ & $2 \times 10^{-6}$ & $2 \times 10^{-6}$ \\
\hline $\mathrm{D}$ & 4.5 & 4.8 \\
\hline $\mathrm{H}$ & 6 & 6 \\
\hline$N$ & $2 \times 10^{10}$ & $1 \times 10^{10}$ \\
\hline$\sigma_{\mathrm{z}} / \mathrm{mm}$ & 0.7 & 5.0 \\
\hline$\beta^{*} / \mathrm{mm}$ & 4 & 15 \\
\hline$\sigma_{\mathrm{t}} / \mu \mathrm{m}$ & 0.30 & 0.55 \\
\hline $\mathrm{E}_{\mathrm{c}} / \mathrm{MeV}$ & 600 & 23 \\
\hline$\Upsilon$ & $1.2 \times 10^{-2}$ & $4.7 \times 10^{-4}$ \\
\hline$\langle\epsilon\rangle$ & $1.7 \times 10^{-2}$ & $1.8 \times 10^{-5}$ \\
\hline $\mathrm{N}_{\gamma}$ & 3.6 & 1.0 \\
\hline $\mathrm{W} / \mathrm{GeV}$ & 100 & 100 \\
\hline$\sigma_{w}$ & $1.1 \times 10^{-2}$ & $2.0 \times 10^{-4}$ \\
\hline$\Delta \mathrm{W} / \mathrm{MeV}$ & 1,100 & $20^{*}$ \\
\hline
\end{tabular}

*) This spread is small enough because $\mathrm{N}_{\gamma}=1$ and in a large fraction of the collisions no photon is radiated. Note that the spread $\Delta W$ can be further reduced proportionally to $\mathrm{L}$. 
than 100 times higher than the one of LEP100. For $Z^{\circ}$ production a large beamstrahlung energy spread ( $\Delta \mathrm{W} \simeq 1 \mathrm{GeV}$, r.m.s) can be accepted. Such a low resolution mode would give $\simeq 10^{9}$ $Z^{\prime}$ s/year, i.e. $\approx 10^{8} \mathrm{~b} \bar{b} /$ year. This rate, larger than what can be obtained at the beauty factory, may be enough to observe some $\mathrm{CP}$-violation effect in the semileptonic decay of $b$-quarks.

A toponium factory would have less luminosity since, if the toponium mass turns out to be in the accessible energy range, one would like to run with $\Delta \mathrm{W} \leq 20 \mathrm{MeV}$. Assuming that the toponium mass is $100 \mathrm{GeV}$, a possible set of parameters providing $\mathrm{L} \simeq 2 \times 10^{32} \mathrm{~cm}^{-2} \mathrm{~s}^{-1}$ is given in the third column of Table 3.

To have a small energy spread both colliders work in the classical regime $(\Upsilon<<1)$ with a small average number of radiated photons, as for the other colliders considered in this paper. The main difference is in the bunch length. For the low resolution $Z^{0}$-factory the bunch length is $\sigma_{z} \simeq 0.5 \mathrm{~mm}$. Such a value is compatible with the very large frequencies considered at CERN for the copper structures powered by a low energy beam whose energy is replenished by superconducting cavities. We underline that the $300 \mathrm{~m}$ of SC cavities used in the second acceleration stage of the bottonium factory (Fig. 4) are exactly what is needed for the driving beam powering a $(500+500)$ meter long copper structure, which would run at $\simeq 100 \mathrm{MV} / \mathrm{m}$ and produce $(50+50) \mathrm{GeV}$ collisions with the quoted luminosities ${ }^{10}$.

For achieving the high energy resolution required for the toponium factory, the bunch has instead to be long $\left(\sigma_{z} \simeq 5 \mathrm{~mm}\right)$ since $\langle\epsilon\rangle$ is proportional to $\sigma_{z}^{-1}$. This requires a long wavelength, i.e. a relatively small frequency $\left(f_{\mathrm{RF}}=350-500 \mathrm{MHz}\right)$. The toponium factory is thus a natural extension of the fully superconducting beauty factory described above. To make it not too long, higher gradients than $7 \mathrm{MV} / \mathrm{m}$ are needed. Here the 'warm' superconductors deposited inside a metallic cavity would find their best use. Of course, once built this collider could be used with short bunches as $Z^{\circ}$-factory with a set of parameters similar to the ones appearing in the second column of Table 3.

Finally, I want to underline that the complex drawn in Fig. 4 is the injector needed for the present CLIC design ${ }^{10,28}$. The electron source and the positron damping rings provide the bunches of the wanted emittance at twice the required repetition frequency. Moreover the $\sim 3 \mathrm{GeV}$ intense electron bunches needed to power the CLIC $30 \mathrm{GHz}$ copper structure can be accelerated by the second racetrack linac.

\section{COST ESTIMATE}

We have made a first rough cost estimate of the accelerator complex schematically drawn in Fig. $4^{23}$. The costs of the DR's is estimated by using $70 \mathrm{kSF} / \mathrm{m}$, which is more than three times the unit cost of the LEP magnet and RF systems: $400 \mathrm{~m} \times 70 \mathrm{kSF} / \mathrm{m} \simeq 28 \mathrm{MSF}$. The costs of the SC cavities and cryostat are also assumed to depend only on length. With $230 \mathrm{kSF} / \mathrm{m}$, as given for LEP 200 cavities by Bernard, Lengeler and Picasso in Ref. 5 , we get $230 \mathrm{kSF} / \mathrm{m} \times 400 \mathrm{~m} \simeq 92 \mathrm{MSF}$. In a reasonable layout (Fig. 4) there are about $500 \mathrm{~m}$ of recirculators. Using half of the unit cost used for the DR's, we get about $18 \mathrm{MSF}$. For the focusing arcs we also take $35 \mathrm{kSF} / \mathrm{m}$ for a total length of $\simeq 200 \mathrm{~m}$, i.e. $7 \mathrm{MSF}$. 
At $500 \mathrm{MHz}$ and $7 \mathrm{MV} / \mathrm{m}\left(\mathrm{R} / \mathrm{Q}=320 \Omega\right.$ and $\left.\mathrm{Q}=4 \times 10^{9}\right)$ the cryogenic losses are $\sim 40 \mathrm{~W} / \mathrm{m}$, i.e. the cryogenic power to be installed is $P_{\text {cryo }}=15 \mathrm{~kW}$ at $4.8 \mathrm{~K}$, which corresponds to about $8 \mathrm{MW}$ at room temperature. The cost ${ }^{5}$ of the cryogenic system is $C_{\text {cryo }}=2.5 \mathrm{MSF}_{\text {cryo }} \approx 1 / 5 \simeq 13 \mathrm{MSF}$.

Finally the cost of the RF-system can be estimated ${ }^{5}$ at the unit price of $1.8 \mathrm{MSF} / \mathrm{MW}$ for a total of $\leq 3 \mathrm{MW}$, i.e. $6 \mathrm{MSF}$.

Summing up the cost of the main components would be about $\approx 165 \mathrm{MSF}$. The electron gun and the positron source (which is certainly very expensive) will bring the material cost of such a collider to about 220 MSF. Manpower, controls, civil engineering and contingency are not included in this estimate. By taking $20 \%$ contingency and 50 MSF for the buildings, one reaches an investment cost of $\sim 320 \mathrm{MSF}$, corresponding to $\sim 300$ Glire for the full complex (no manpower included). These figures have to be taken only as first order indications.

\section{CONCLUSIONS}

I have described the conceptual design and the staging possibilities of an accelerator complex based on SC radiofrequency cavities. Before concluding two remarks are in order. First, the accelerator complex offers the unique possibility of bringing back relevant particle physics to a medium size laboratory and, at the same time, opens a lot of other avenues. This is a guarantee of long life for the laboratory, but does not imply that all the options should be followed in the order I presented them. I have presented a menu; the constructors will have to choose, at the proper moment, what to do. Second remark: the presentation has been mainly technical because the interesting diverse physics issues, which could be tackled with such a facility, can be read in many good reviews and to be even cursorily listed would require much more space. This asymmetry in the presentation should not leave the reader with the false impression that the reason for building such a complex is technical: the program finds its justification in the fact that important questions in the physics of moleculae, nuclei, heavy flavours, fundamental interactions, etc., can be addressed and answered. Moreover, the laboratory would also contribute useful technical developments for both future accelerators and industrial applications (for instance in the field of power millimeter FEL for fusion reactors). In particular, this would be perfectly in line with the policy of the Italian Institute for Nuclear Research (INFN), which, in the last five year plan, pointed to advanced technology in the field of superconductivity.

A conceptual design is not a proposal. To move in this direction a first meeting was held in Frascati (April 22nd and 23rd, 1987) and now working groups should be set up to look into the different parts of the SC radiofrequency complex. The groups should review the design and also give a price tag and a time scale to the various stages. This, I believe, could be done by the beginning of 1988.

As far as the realization is concerned, I think that such a complex can be built in Italy, but that an international participation is needed not only to share some of the expenses (in particular for the detectors, which have not been discussed at all) but also to use the competences which exist in many European laboratories. 


\section{ACKNOWLEDGEMENT}

I am grateful to Sergio Tazzari for the many illuminating discussions we have had since July 1985 on the many facets of this subject and for useful suggestions on the content of this paper. Most of the work reported here has been done together with Guy Coignet, in a most pleasant spirit of friendship and collaboration. The Yokoya computer simulation program has been run by P.T. Cox, whom I thank very warmly.

\section{REFERENCES AND NOTES}

1) R. Sundelin, IEEE NS-32 (1985) 3570.

H. Piel, IEEE NS-32 (1985) 3565.

2) H. Lengeler, Proc of the 13th Int. Conf. of High-Energy Acc., Novosibirsk, 7-11 August 1986 and CERN/EF/86-15 (July 1986).

3) D.A.G. Deacon et al., Phys. Rev. Letters 38 (1977) 892.

4) CEBAF Design Report, May 1986. W. Bensiek et al., in Proc 1986 Lin. Acc. Conf., Stanford, U.S.A.

J. Mougey, Proc of the European Workshop on Few-Body Physics, Rome, 7-11 Oct. 1986, C. Ciofi degli Atti, O. Benhar, E. Pace and G. Salmè Eds., Springer-Verlag, Wien and New York, 1986, p. 571.

5) R.M. Sundelin et al., Proc 9th Int. Conf. High En. Acc., 1974, 128.

P. Bernard, H. Lengeler and E. Picasso, CERN/LEP-Di/8629 and Contribution to the Workshop LEP 200, Aachen, Sept. 29-Oct. 1, 1986.

6) M. Tigner, Nuovo Cim. 37 (1965) 1228

7) U. Amaldi, Phys. Lett. B61 (1976) 313.

8) D.H. Rice, Cornell University, CLNS-85/708 (November 1985).

R. Sundelin, Cornell University, CLNS-85/709 (November 1985).

H. Padamsee, Cornell University, CLNS-85/710 (November 1985).

9) U. Amaldi, H. Lengeler and H. Piel, CERN EF 86-8 (May 1986) and CLIC Note 15.

10) W. Schnell, LEP-RF/WS/PS (1985) and CLIC Note 13 (1986).

11) U. Amaldi and C. Pellegrini, CLIC Note 16 (1986), to be published in the Proc of the Workshop on Future Accelerators, Erice, 1986.

12) V. Lagomarsino et al., IEEE Trans. MAG-15 (1979) 25 ,

P. Kneisel et al., Nucl. Instr. Meth. 188 (1981) 669.

13) C.W. Leeman, The continuous electron beam accelerator facility (CEBAF), Ref. 24, p. 125.

14) C. Benvenuti et al., Appl. Phys. Lett. 45 (1984) 583.

C. Benvenuti et al., Production of SC RF cavities by coating copper with a sputtered layer of niobium, to be presented to the Workshop on High $T_{c}$ Superconductors and Potential Applications, Genova, July 1987.

15) I.E. Campisi, On the limiting RF fields in SC accelerator cavities, SLAC/AP-58 (April 1987).

16) The participants to the round table discussion were: R.A. Ricci (Chairman), U. Amaldi, M. Hawak, C. Rubbia, A. Salam and A. Zichichi.

17) U. Amaldi, A toponium factory on the way to TeV electron-positron linear colliders, CERN Accelerator School, Oxford, 26 Sept 1985.

18) C. Albajar et al., UA1 Collaboration, Phys. Letters B, 186 (1987) 247.

M. Albrecht et al., Argus Collaboration, DESY 87-029 and Phys. Letters, in print. 
19) See the Proc of the Int. Symposium on Production and Decay of Heavy Hadrons, Heidelberg, May 20-27, 1986, K.R. Schubert and R. Waldi Eds.

20) R. Eichler, T. Nakada, K.R. Schubert, S. Weseler and K. Wille, Motivation and design study of a B-meson factory with high luminosity, SIN,PR-86-13, (November 1986).

21) $U$. Amaldi and $G$. Coignet 'An electron-positron linear collider as a $B-\bar{B}$ meson factory', LAPP-EXP-86-71 (July 1986).

22) U. Amaldi and G. Coignet, Proc. of the Symposium on Critical Issues in Development of New Linear Colliders, Madison Wisconsin, August 1986 (to be published).

G. Coignet, Proc. of the B-B Workshop, UCLA, Los Angeles, Jan. 1987,D. Cline Ed. (to be published).

23) U. Amaldi and G. Coignet, Conceptual design of a multipurpose beautyfactory based on SC cavities, CERN-EP/86-211 and Nucl. Instr. Methods (in print).

24) The Discussion Meeting on SC Linear Accelerators, Frascati, October 13-14, 1986, was organized by U. Amaldi, C. Guaraldo, C. Pellegrini,A. Renieri, S. Tazzari (Chairman), F. Tazzioli. It was sponsored byINFN and ENEA. The proceedings were edited by S. Stipcich.

25) T.I. Smith, Nucl. Instr. Meth. A250 (1986) 64.

26) R. Sheffield, Photocathodes in accelerator applications, Workshop on Low Emittance Beams, Brookhaven National Lab., March 20-25, 1987.

J.S. Fraser, Photocathodes in accelerator applications, Proc. of the 1987 Acc. Conf., Washington, DC, March 16-19, 1987 and LA-UR 87-863.

27) J. Kirkby, A tau-charm factory at CERN: precise experiments to confront the standard model, CERN/EP Internal Note, (May 1987).

28) Report of the Advisory Panel on the prospects for $\mathrm{e}^{+} \mathrm{e}^{-}$linear colliders in the TeV range, submitted to the CERN Long Range Planning Committee chaired by C. Rubbia, CLIC Note 38 , (May 1987).

29) T. Himel and J. Siegrist, SLAC-PUB-3572 (February 1985).

R. Nobel, AAS-note, SLAC (1985).

30) K. Yokoya, Nucl. Instr. Meth. A251 (1986) 1, and KEK Report 85-09 (1985).

31) U. Amaldi, Nucl. Instr. Meth. A243 (1986) 312 and CLIC Note 2.

32) R. Holleebeck, Nucl. Instr. Meth. 184 (1981) 333.

33) SLC Design Handbook, Stanford Linear Accelerator Center (December 1984).

34) R. Chasman and K. Green, Brookh. Nat. Lab. Report BNL 50505 (1980).

35) K. Brown, private communication. 